\title{
Photonic Crystal Fibers for Sensing Applications
}

\section{Boni Amin Riyadh SM*, Md. Mahbub Hossain, Himadri Sekhar Mondal, Md. Ekhlasur Rahaman, Pankoj Kumar Mondal and Md. Mehadi} Hasan Mahasin

Electronics and Communication Engineering, Khulna University, Khulna, Bangladesh

\begin{abstract}
This article reviews the recent progress in optical sensors using photonic crystal fiber (PCF) technology, which is newly adopted beyond conventional optical fibers (OFs) due to their unique geometric structures. PCFs have some exceptional properties. It will focus the most conversant sensing application areas with their consistent parameters and perception. Numerous PCF sensors are available in day-to-day world. Researchers and technologists are working on PCFs with more than 15-20 application areas. In this article, sensing applications in physical and biochemical with their diverse types are reviewed recent times. Physical sensors as temperature, curvature, torsion, vibration, pressure, refractive index, evanescent; where the bio-chemical sensors as humidity, gas, $\mathrm{pH}$, liquid etc. has been reviewed in this article. The review shows that, most physical sensing applications are varied with their different structures and parameters.
\end{abstract}

Keywords: Photonic crystal fiber; Optical sensor; Physical sensor; Biosensor

\section{Introduction}

Photonic crystal fiber (PCF) is a kind of optical fiber (OF) that uses photonic crystals to form the cladding around the core of the cable. Photonic crystal is a low-loss periodic dielectric medium constructed using a periodic array of microscopic air holes that run along the entire fiber length. In PCFs, photonic crystals with photonic band gaps (PBG) are constructed to prevent light propagation in certain directions with a certain range of wavelengths. Contrary to normal fiber optics, PCFs use total internal reflection or light confinement in hollow core methods to propagate light. Light propagation in PCFs is far superior to standard fiber, which uses constant lower refractive index cladding. Applications for photonic crystal fibers include spectroscopy, metrology, biomedicine, imaging, telecommunication, industrial machining and military technology. Photonic crystal fiber is also known as micro structured, or holey, fiber [1-5].

OF evolution in 1966 changed fields such as telecommunication and sensing, leading to the inception of high sensitivity and restrained systems based on light conveyance. This phenomenal innate of fiber optics such as geometric adaptability, increased sensitivity over extant techniques, and inherent amity with fiber optic communications technology make them stand out for sensing applications. The advantages of PCF over conventional OF depends on size, modelling, band solving and different parametric properties. As an example, a temperature sensor differ the parametric properties due to its size and geometry. OF doesn't need any geometrical phenomena and thus it's based on normal operation [6-10].

In this article, numerous sensors are described according to their parameters and sensitivity.

The lists of current PCF sensors in world are categorized below (Figure 1):

According to core structure, P CFs are of two types: (i) Solid Core (SC) and (ii) Hollow Core (HC). PCFs are basically constructed with periodic air holes and for this reason; Refractive Index (RI) varies with the change in pitch. The application areas of SC are: high power handling, fiber laser multi-wavelength generation, long period gratings, optical coherence tomography and spectroscopy [11-15].

In this article, different types of sensors are reviewed according to their sensitivity parameters. The basic ideas and examples of current trend can be analyzed from this article into other future researches later. As there are many structural performance of PCF sensors diverge from one another, a large number of diverse structures can be assembled. PCF sensors have enormous criteria over OFs, consequently this critique can facilitate the researchers or scientists further [16,17].

\section{Photonic Crystal Fiber (PCF) Structures}

Most Photonic Crystal Fiber (PCF) has geometrical structure in their formation. The structures of the adjacent array holes are mostly tetragonal, hexagonal, octagonal, quadrilateral, pyramid cal, Lissajou's pattern and many more. Conventional PCF structures of recent trend with their short representation are described in the following sub sections hereby $[18,19]$.

\section{Hexagonal holes pattern}

These types of PCFs are used in temperature, refractive index (RI), molecular and evanescent sensors in general. Here the array of periodic holes makes a hexagonal pattern as anticipation. These holes can be air, solid or air with metallic boundary types. The diameter and pitch is different in a single PCF sometimes. The following is an example of a hexagonal PCF sensor (Figure 2).

Here the $d_{\text {hole }}$ and $\Lambda$ denote diameter of holes, diameter of core and pitch, respectively. The holes are air holes and it can be any other dielectrics.

\section{Hexagonal holes with void channel}

It can also be made a hexagonal PCF described in the earlier subsection with a void or empty channel where the photons can easily

*Corresponding author: Boni Amin Riyadh SM, Electronics and Communication Engineering, Khulna University, Khulna, Bangladesh, Tel: 8801701491184; E-mail: boni.ku03@gmail.com

Received February 02, 2018; Accepted February 20, 2018; Published February 27, 2018

Citation: Riyadh SMBA, Hossain MM, Mondal HS, Rahaman ME, Mondal PK, et al. (2018) Photonic Crystal Fibers for Sensing Applications. J Biosens Bioelectron 9: 251. doi: 10.4172/2155-6210.1000251

Copyright: ( 2018 Riyadh SMBA, et al. This is an open-access article distributed under the terms of the Creative Commons Attribution License, which permits unrestricted use, distribution, and reproduction in any medium, provided the original author and source are credited. 


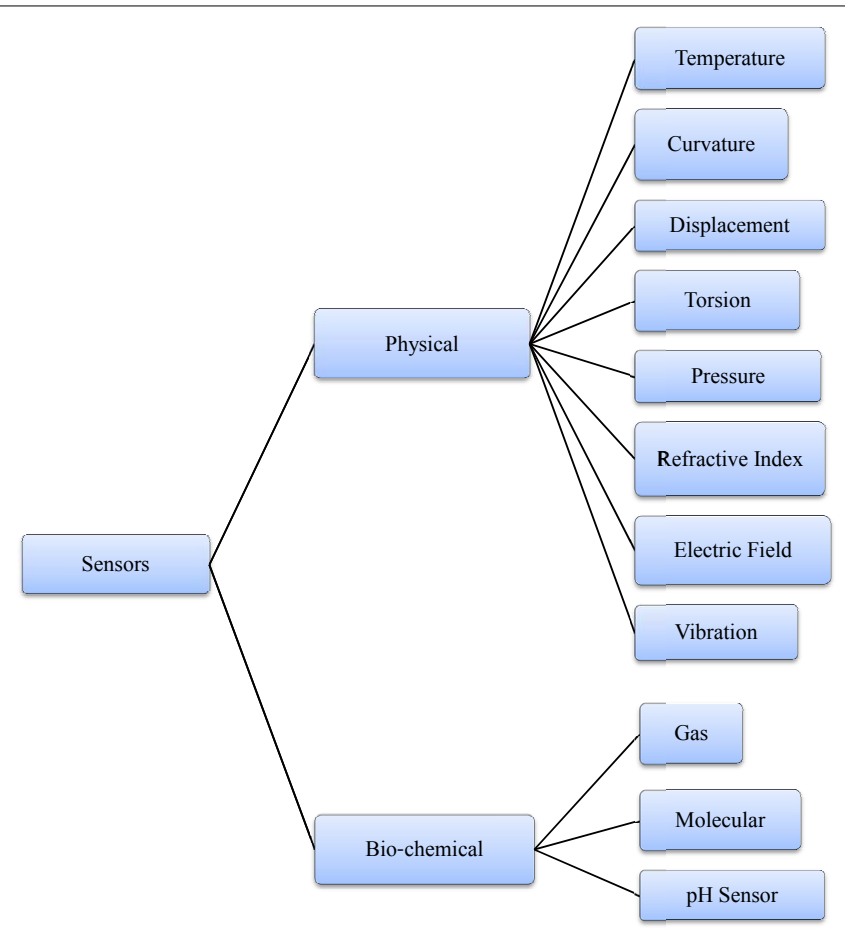

Figure 1: Categorization of current PCF sensors in world.

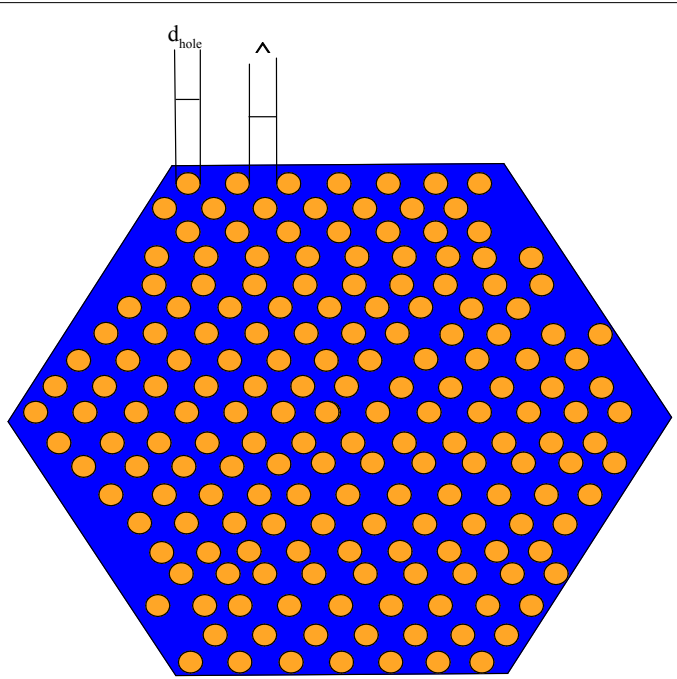

Figure 2: An example of a hexagonal PCF sensor.

penetrate. The formation and materials of the structures are similar to the previous structure. The void region can be different types of pattern like English letters, numbers, butterfly, flower petal etc. Generally, in Mach-Zehnder PCF structure, a letter " $Z$ " shape is used versatile. The shape can be depicted as follows (Figure 3).

The black line through the void channel indicates the photon or light energy penetration. The yellow holes can be air or any other dielectrics.

\section{Circular/oval shape structures}

Numerous temperature sensors are made of circular shaped PCF. Some pressure and bio-sensors also are made of these types of shape.

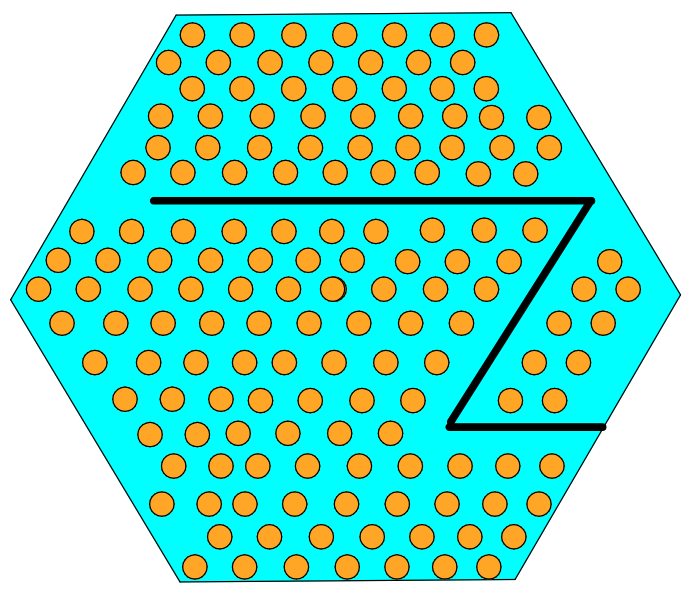

Figure 3: Hexagonal holes with void channel.

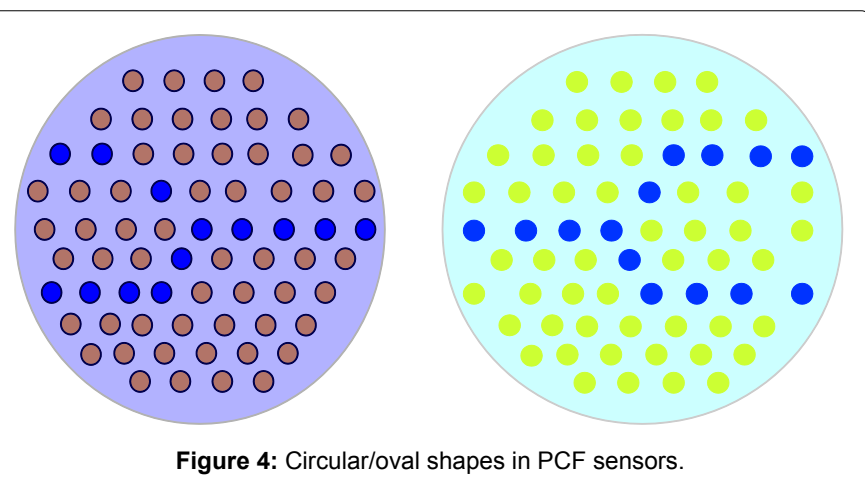

The difference between hexagonal and circular is according to their special geometric patterning. It can be easily figure out the trending circular shape in PCF sensors in following structures (Figure 4).

Two types of colour are seen here to denoting the dielectric or material differentiation. In the leftmost picture, blue holes are solid holes and the rest are air holes. In the rightmost picture, the blue holes are air where the rests are solid holes. Both are in horizontal "Y" shape which is familiar in Mach-Zehnder PCF.

Another circular or ellipse type shapes are on research recent days. These types of PCF are vastly used in evanescent sensors, electrical field sensor, $\mathrm{pH}$ sensor, velocity sensor etc. The key parameters in these sensors are: they have several mismatched holes in diameter. A centre hollow core can be larger than all other holes on that PCF and vice versa. The hollow core can be replaced by solid core as well as the change in category. The reflection in changing the hollow core and solid core enhances the field of PCF sensor. The following is some current version of PCF sensors (Figure 5).

Here in this picture (left), the cyan holes are hollow core where the blue holes are solid. Three different types of dielectric are operational here: air, solid and substrate. Similar to this type, Sagittarius shape PCF can be constructed (right).

\section{Octagonal shape structures}

Despite of many shapes, octagonal shapes have immense popularity in recent researches because of their geometric pat terns (Figure 6).

Here, blue holes are solid, centre white hole is hollow core or air 

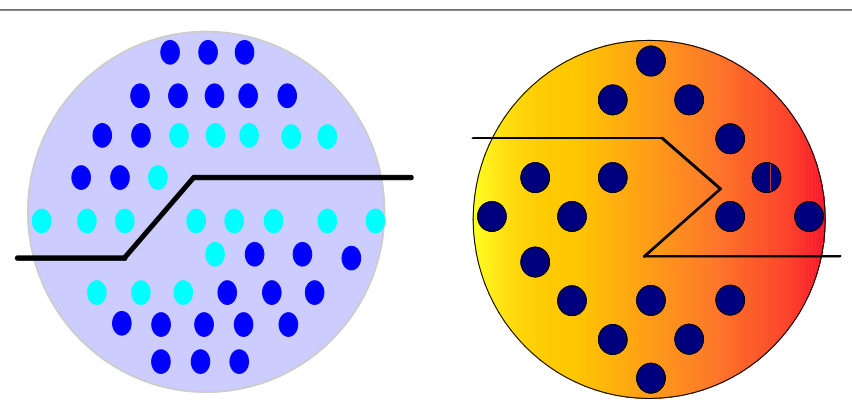

Figure 5: Current version of PCF sensors.

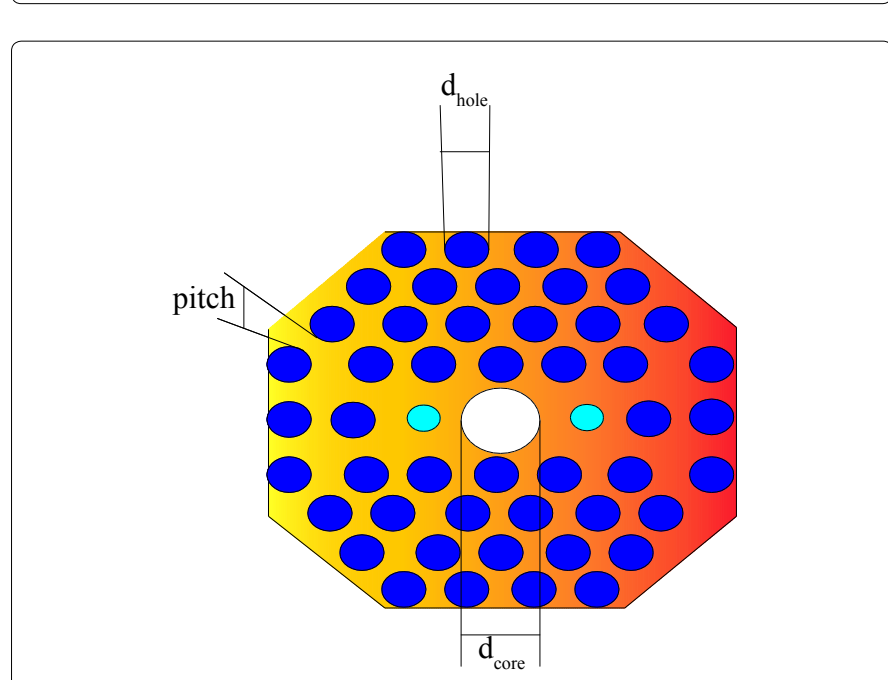

Figure 6: Octagonal shape structures.
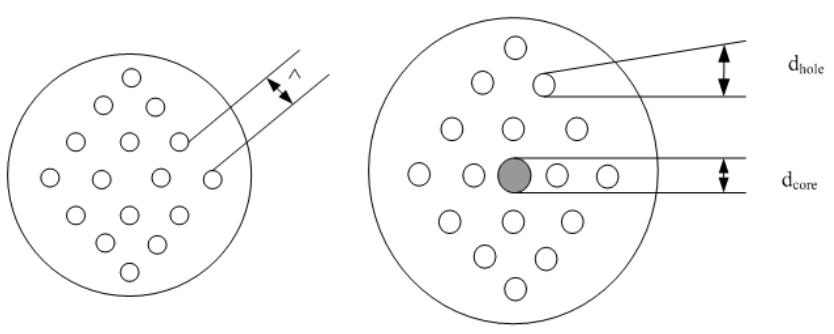

Figure 7: Solid-core and hollow core PCFs (left to right).

core, and tiny cyan holes are of different dielectric. This type of PCF sensor is basically used in electrical field sensor, $\mathrm{pH}$ sensor, food processing sensor and bio-sensors.

Solid core PCFs has the core with organic, metal or metal-organic material cores. The core consists of dielectric constants parameter here. Similarly, for hollow core-it consists of a void air hole and the calculation of Sellmeier equation is easier. Figure 7 shows both types of fiber in the following [14].

Here the $\mathrm{d}_{\text {hole }}, \mathrm{d}_{\text {core }}$ and $\Lambda$ denote diameter of holes, diameter of core and pitch, respectively.

\section{PCF Classification as a Sensor}

Sensing applications regarding PCF are versatile and drastically used in modern days. Physical, biochemical and biological sensors are used for different features. Physical sensors are temperature, curvature, displacement, torsion, pressure, refractive indices, electrical field, and vibration sensors. Biochemical sensors that are mostly familiar to us are gas, molecular, pH sensor etc. Environment, food processing, food monitoring, glucose sensors are recently added into these fields. In this article, these PCF sensors have been reviewed: biosensor, curvature, evanescent, gas, humidity, liquid, $\mathrm{pH}$, Plasmon, pressure, refractive index, temperature, torsion, vibration etc. Every form of PCF sensor has its intrinsic parameters but sensitivity dissents from one another, which will be delineated forth. A concise prominence on sensors can help to perceive the functions and applications herewith [20-25].

\section{Biosensors}

It implements a new aperture for diverse promising applications including biological sample tracking, antibody-antigen collaboration, medical diagnosis, organic-chemical sensing, food quality control, bio imaging, environment monitoring, and so on. Generally bio sensors using PCF are a two layer circular lattice structured with gold layer thickness of $40 \mathrm{~nm}$ and maximum sensitivity $2200 \mathrm{~nm} / \mathrm{RIU}$. Using simple amplitude interrogation method, biosensors have the maximum sensitivity as $266 \mathrm{RIU}^{-1}$ and resolution $3.75 \times 10^{-5} \mathrm{RIU}$ [22].

\section{Curvature}

Curvature, bending, and strain of engineering structures are relevant parameters meticulously associated with the mechanical loading and health condition of the structures. A lot of sensors based on PCF have been designed for bending or strain calibration, such as Long period Grating (LPG), FBG, and Mach-Zehnder interferometers, etc. The curvature sensor structure based on a Mach-Zehnder interferometer (MZI) usually mires two photonic mode-coupling sections, where the two sections perform as a splitter and a combiner. Sandwiched PCF has the sensitivity $60.3 \mathrm{pm} /{ }^{\circ} \mathrm{C}$ in MZI wavelength and $9.2 \mathrm{pm} /{ }^{\circ} \mathrm{C}$ in Bragg wavelength. Curvature sensitivity varies $-0.003 \mathrm{~nm} / \mathrm{m}^{-1}$ in the range of $10 \mathrm{~m}^{-1}$ to $22.4 \mathrm{~m}^{-1}$ wavelength nowadays $[5,7]$.

\section{Evanescent}

The evanescent field affiliated with the light propagating in the confinement region of the device extends into the section where the analyte to be sensed is located. The existence of air holes in the cladding microstructure allows the compliance of biological and chemical samples in gaseous or liquid forms in the extant locality of the PCF core. Air hole elliptical PCF with V-shaped and $\mathrm{H}$-shaped are familiar to recent trending. [2,19].

\section{Gas}

Gas sensors based on hollow-core PBGPCFs are proposed in to confine more light in the core, which incorporates the gas sample. At present, most of the gases sensing techniques tend to be substantial, costly, and lack acute time data measurement; thus, surrogate sensing methods are in great demand. Some different lattice structures like octagonal, hexagonal, decagonal, circular honeycomb cladding, elliptical are the better guiding properties of PCF. Increasing diameter of air hole and decreasing pitch, relative sensitivity enhanced properly. The highest relative sensitivity in a gas sensor can be $42.27 \%$ with confinement loss of $4.78 \times 10^{-6} \mathrm{~dB} / \mathrm{m}$. For slotted core and hexagonal substrate, $48.26 \%$ relative sensitivity with birefringence of $2.17 \times 10^{-2}$ is experimented in researches [18-25].

\section{Humidity}

PCF humidity sensors (PCFHSs) offer several facilities over electronic humidity sensors such as micro design, persistence, the 
possibility of working on flammable environments and at high temperature and pressure panoramas, and, most important, their electromagnetic immunity. Therefore, they can dispute the kind of harsh and demanding conditions found in industrial areas. High sensitivity in low complexity is the key promoter for humidity sensor. Here Bragg grating resonators are used versatile [24].

\section{Liquid}

Highly sensitive chemical (liquid and gas) sensors are playing a salient role in the industrial processes especially for detecting toxic and combustible chemicals (e.g., toxic gases or liquids) to swamp the safety issues. Therefore, it has become one of the key challenges to intense the performance of liquid and gas sensors. Nowadays, researchers are keeping much concern on the development of PCF based sensors for environmental and safety monitoring issues. PCF based liquid and gas sensors through the evanescent field show peerless performance in terms of sensitivity, because core of the PCF directly relates with the material to be analysed. Hexagonal H-PCF with low confinement loss is used in vivid areas of liquid sensor applications. The so far experiment defines that the higher sensitivity can be conducted by increasing diameter of innermost ring air hole in the substrate. Also by placing a single channel instead of group of tiny channels, increases sensitivity more [26-28].

\section{pH}

There are several methods of $\mathrm{pH}$ measurement developed viz. $\mathrm{pH}$ test strips, $\mathrm{pH}$ glass microelectrodes, indicator reagents, and other potentiometric devices to name a few. However, compared to traditional pH detective methods, PCF sensor is more appealing in view of its small size, electromagnetically immune, faster response and has the capability for multiplexing and remote sensing. Exponential decay of evanescent wave at core-substrate interface is determined to $\mathrm{pH}$ sensing. The intensity is measured using SPSS [23].

\section{Plasmon}

In particular, SPR sensors offer many advantages such as high sensitivity as well as label-free, high sensitivity and rapid real-time detection. Because of the high sensitivity to small variations in the refractive index of the probed medium, small concentrations of bio molecules can be detected. In the stereotyped Kretschmann SPR configuration, a coupling prism with a thin metal-deposited base is used, and in the ubiquity of incident light at a specific angle, SPs will be excited on the metal-dielectric interface. However, the orthodox SPR configuration is bulky and not expedient for remote sensing, which limits its large-scale fabrication for real time applications. PCF-SPR sensor with selectively metal-coated air holes that obtained a sensitivity of $5500 \mathrm{~nm} / \mathrm{RIU}$ has been experimented earlier. Amplitude sensitivity of $4398 \mathrm{~dB} / \mathrm{RIU}$ with resolution $2.27 \times 10^{-6} \mathrm{RIU}$ has been seen recently. To prevent oxidization, silver coated grapheme layer is initialized with Leaky-Gaussian core guided mode [9].

\section{Pressure}

This sensor is based on the air holes type structure, which is more preferable in nano-electromechanical application systems. T. Stomeoet designed and fabricated a 2D photonic crystal. It is strain sensitive. This sensor is also based on the resonance wavelength shift. It detects the pressure from $0.25 \mathrm{Gpa}$ to $5 \mathrm{Gpa}$ and its sensitivity is $5.18 \mathrm{~nm} / \mathrm{Gpa}$. The Sagnac loop itself acts as a sensitive pressure-sensing element, making it an ultimate candidate for pressure sensor. Other reported PCF pressure sensors usually required some sort of adaptation to the substrate to boost their sensitivity. Higher birefringence PCF with fast sensitivity of $8.97 \times 10^{-6} \mathrm{RIU} / \mathrm{MPa}$ is recent trending $[10,13]$.

\section{Refractive index}

In recent years, numerous PCF based RI sensors based on modal interferometry, Sagnac loop, ring resonator, tapered channel have been proposed. RI sensors based on modal interferometry use the intrusion of core and substrate mode while those based resonant uses the interference of two coherent mechanism of a source in a PCF in case of ring resonator. Basically 1.05 1.10 RI can be measured using RI sensors. As the wavelength decreases, RI sensitivity increases linearly $[6,11,12]$.

\section{Temperature}

Temperature PCF sensors have applications in various fields such as medicine/biomedicine, the food and beverage industry, agriculture, industrial processing, and research. In the field of medicine, PCF sensors are used in real time structural health monitoring (SHM), organ transplant systems, kidney dialysis machines, and medical incubators. In the agricultural sector, they are used to monitor the temperature of plants, soil, and water. In the food and beverage industry, PCF sensors are used in fermentation, brewing, meat processing, and the manufacture of storeroom tanks. PCF temperature sensors are also versatile in the petro-chemical industry, the automotive industry, metal industries, geo-thermal wells, electronics industry, petroleum industries, and in harsh environmental applications. Blue shift occurs when temperature decreases and the birefringence is low here. High sensitivity occurs with spectral wavelength and the refractive index should be more than 1.4 in these cases. At this refractive index, average sensitivity can be $43400 \mathrm{~nm} / \mathrm{RIU}$ which can be enhanced to $77000 \mathrm{~nm} /$ RIU [1-4,8,26,27].

\section{Torsion}

The measurement of torsion is an imperative method for the inservice evaluation and monitoring condition of engineering structures such as bridges, buildings, trains and so on. In general, the PCF torsion sensors are separable into two classes: the PCF bragg-grating based torsion sensors and the interferometer-based torsion sensors. Torsion was also measured by a $16 \mathrm{~mm}$-long period grating channel with engraved substrate and a twist sensitivity of $6.279 \times 10^{-4} \mathrm{~nm} /{ }^{\circ}$ was achieved $[15,16]$.

\section{Vibration}

In the field of structural health monitoring, seismic activity, infringement detection, and many other applications, standard accelerometers are widely used as the most proficient sensing systems for vibration measurements. These PCF sensors are composed of a structure of holes surrounding a concrete core, which offers a unique design flexibility to optimize their waveguide properties for unambiguous applications. Butterfly structures are used generally with two orthogonally polarized fields. $50 \mathrm{~Hz} \sim 1 \mathrm{kHz}$ range is temperature insensitive [17].

\section{Theoretical Background}

Different types of sensor have diverse strategy to analyse the performance. Each sensor has different sensitivity and equations. As stated above in the previous section, the sequences of sensitivities are as follows.

For a biosensor, it generally uses the Sellmeier equation to calculate the effective refractive index $\mathrm{n}$ with respect to wavelength $\lambda$. 


$$
n^{2}(\lambda)=1+\frac{B_{1} \lambda^{2}}{\lambda^{2}-C_{1}}+\frac{B_{2} \lambda^{2}}{\lambda^{2}-C_{2}}+\frac{B_{3} \lambda^{2}}{\lambda^{2}-C_{3}}
$$

Here B,C are arbitrary constants that depends on core material. The confinement loss can be measured by the following equation.

$$
=8.868 k_{0} \operatorname{Im}\left(n_{e f f}\right)
$$

Here the term $k_{0}$ denotes wavelength function $(2 \pi / \lambda)$. The sensitivity of a biosensor depends on the change of wavelength and refractive indices. The ratio of peak wavelength change to refractive index is known as the sensitivity. Therefore the unit of these sensors must be $\mathrm{nm} / \mathrm{RIU}$. The sensitivity is basically figured as $S_{\lambda}$.

$$
\mathrm{S}_{\lambda}(\mathrm{nm} / \mathrm{RIU})=\frac{\Delta \lambda \text { peak }}{\Delta \mathrm{n}_{\mathrm{a}}}
$$

Sometimes it is needed to calculate the resolution and amplitude sensitivity to increase efficiency and application areas. Hence, the corresponding equations should be,

$$
\begin{aligned}
& \text { Resolution, } \mathrm{R}(\mathrm{RIU})=\frac{\Delta n_{a} \Delta \lambda_{\min }}{\Delta \lambda_{\text {peak }}} \\
& \text { Amplitude sensitivity, } \mathrm{S}_{\mathrm{A}}\left[\mathrm{RIU}^{-1}\right]=-\frac{1}{\alpha\left(\lambda, n_{a}\right)} \frac{\partial \alpha\left(\lambda, n_{a}\right)}{\partial n_{a}}
\end{aligned}
$$

The resolution and amplitude sensitivity are inversely related to each other for biosensors [22].

Curvature sensing can be measured by altering the curvature (C), bending displacement $(\mathrm{h})$, half distance between MMF (d), curvature difference $(\Delta \mathrm{C})$ and temperature difference $(\Delta \mathrm{T})$.

$$
\begin{aligned}
& \text { Curvature, } \mathrm{C}=\frac{2 h}{h^{2}+d^{2}} \\
& \text { Curvature radius, } \rho=\frac{1}{C}=\left[\rho^{2} \cos ^{2}\left(\frac{D}{\rho}\right)+d^{2}\right]^{0.5} \\
& C=\frac{\sqrt{3\left(D^{2}-d^{2}\right)}}{D^{2}} \\
& {\left[\begin{array}{l}
\Delta T \\
\Delta C
\end{array}\right]=\left[\begin{array}{ll}
-0.32 & 110.81 \\
-0.99 & 6.49
\end{array}\right]\left[\begin{array}{l}
\Delta \lambda_{1} \\
\Delta \lambda 2
\end{array}\right]}
\end{aligned}
$$

Eqn. (9) is the matrix representations of two different equations which follows the curvature criteria [5]. Curvature radius can be measured according to eqns. (6) to (10), for different structures. Sometimes, intensity should be evaluated to better optimization of a curvature sensor. The intensity [7] equation depends on curvature angle and is followed,

$$
I=I_{C O}+I_{C 1}+2 \sqrt{I_{C O} I_{C 1} \cos \varphi}
$$

Evanescent sensors mostly depend on their refractive indices, relative sensitivity coefficient and effective refractive index of air holes. Relative sensitivity coefficient ( $r$ ) is directly proportional to the sensitivity with a nominal value of unit less constant.

$$
\begin{aligned}
& r=\frac{n_{r}}{n_{e}} f \\
& \mathrm{f}=\frac{\text { sample } \int \operatorname{Re}\left(E_{X} H_{Y}^{*}-E_{Y} H_{X}^{*}\right) d X d Y}{\text { total } \int \operatorname{Re}\left(E_{X} H_{Y}^{*}-E_{Y} H_{X}^{*}\right) d X d Y} \times 100
\end{aligned}
$$

Here, $\mathrm{f}$ is the sensitivity and measured in percentage. As the EM field alters then the percentage also differs from one another. Also, as the sampling rate falls, sensitivity drops. The terms $n_{r}$ and $n_{e}$ are the refractive indices of material and air holes, respectively [2].

Severe gas sensors are experimented earlier with respect to their substrate effective area, fiber length, concentration of gaseous volume and intensity. It also uses eqn. (13) in maximum usages. Although these have some other concerns below,

$$
\text { Effective area, } \mathrm{A}_{e f f}=\frac{\left(\iint|E|^{2} d x d y\right)^{2}}{\iint|E|^{4} d x d y}
$$

Here another term exists as effective area of gaseous materials or the substrate where it flows.

The effective area can be intended using rudimentary mathematics and complex variable functions. Confinement loss can be reduced by changing the structural parameters and materials. A mutual single mode fiber (SMF) and photonic crystal fiber (PCF) has the relation according to their losses are,

$$
L_{S}=-20 \log _{10} \frac{2 W_{S M F} W_{P C F}}{W_{S M F}^{2} W_{P C F}^{2}}
$$

Here the letter $\mathrm{W}$ denotes the width and $\mathrm{L}_{\mathrm{S}}$ is as substrate confinement loss. [25]

Similar to evanescent sensors, Plasmon sensors follow the identical sensitivity and resolution as eqns. (2) and (3) to (5). A huge number of Plasmon sensor sensitivities are calculated using statistical analyses and procedures. Regression line equation of various Plasmon sensors on $\mathrm{XY}$ axes evaluated earlier as,

$$
\mathrm{Y}=2390 \mathrm{X}-2446(\mathrm{~nm})
$$

Here the $\mathrm{Y}$ axis represents the wavelength due to the alteration of sensitivity at $\mathrm{X}$ axis. The wavelength measured here is apparently in nanometer range. Numerical values like 2390 and 2446 are defined values for Plasmonic coefficients [14].

Despite of above complexities, the Pressure sensors play a major role in mechanical and opto-mechanics. The equations are much easier here to realize and functioning smoothly under heavy load. Pressure sensors are used in giga Pascal scaling. The refractive indices, Poisson's ratio, Young's modulus and phase shifting influences the sensor in most cases. High Birefringence PCF has the pressure variation from 0-100 Mpa. The sensitivity of PCF pressure sensor worth consequent equations,

$$
\begin{aligned}
& \mathrm{n}=\mathrm{n}_{0}-\left(\mathrm{C}_{1}+2 \mathrm{C}_{2}\right) \sigma \\
& \mathrm{C}_{1}=\mathrm{n}_{0}\left(\mathrm{P}_{11}-2 \mathrm{~V} \cdot \mathrm{P}_{12}\right) / 2 \mathrm{E} \\
& \mathrm{C}_{2}=\mathrm{n}_{0}{ }^{3}\left[\mathrm{P}_{12}-\mathrm{V}\left(\mathrm{P}_{11}+\mathrm{P}_{12}\right)\right] / 2 \mathrm{E} \\
& \mathrm{I}_{\mathrm{R}}=2 \mathrm{E}_{0}^{2}\left[1+\cos \left(\mathrm{k}_{0} \mathrm{LB}\right)\right] \\
& \mathrm{B}=\left|\mathrm{n}_{\mathrm{x}}-\mathrm{n}_{\mathrm{y}}\right| \\
& \mathrm{V}=\left[1+\cos \left(\mathrm{k}_{0} \mathrm{LB}\right)\right] / 2
\end{aligned}
$$

Numerous parameters exist into the equations stated above which indicates refractive indices (n), Poisson's ratio (P), Young's modulus (E), refractive indices shift (B), visibility (V) and intensity (I). All equations are linear from eqns. (17) and (18) and eqns. (20) and (22) are sinusoidal. It implies that the arbitrary constants are linear where the intensity is sinusoidal. In addition, the visibility is cosine function. As the wavelength of (20) decreases, intensity boosts. Moreover, the wavelength shrinking results less visibility at device ends. [10,13] 
Refractive index sensor has an extensive application area in optoelectronics and mechanics. Large mode area PCF that are spliced between single mode fibers with low temperature and appears good resolution level. Scanty sampling come off deficient sensitivity hence voluminous sampling is conducted in these sensors. Radius, fiber length, atomic density, dielectric constant and wavelength have to be in account with these studies.

$$
\begin{aligned}
& \mathrm{n}_{\mathrm{eq}}(\mathrm{X}, \mathrm{Y})=\mathrm{n}(\mathrm{X}, \mathrm{Y}) \exp \left[\frac{\left(d^{2}+L^{2}\right) X}{2 d}\right] \\
& \Delta \omega=-\frac{\omega}{2} \frac{\text { sample } \int d^{3} r \Delta \varepsilon(r)|E(r)|^{2}}{\text { total } \int d^{3} r \Delta \varepsilon(r)|E(r)|^{2}}
\end{aligned}
$$

Half distance $(\mathrm{d})$, PCF length $(\mathrm{L})$, frequency $(\omega)$, cavity radii $(\mathrm{r})$ and sample dielectric constant $(\Delta \varepsilon)$ are the major criteria to design the structure of a RI PCF. According to mathematical statement in equation (24), it is precise that the sensitivity resemblances with electromagnetic field, sampling frequency and dielectric constants. The ratio and relation has been patently exhibited here $[6,11]$.

Usually PCF sensing applications infatuate modern photonics technology over temperature sensitivity at large. Photonic Bragg Crystal (PBG) structures are often used in such cases. The deviation in wavelength and effective refractive index causes dispersion and temperature sensitivity. At basal temperature, birefringence is low scarcely thus, high sensitivity occurs with spectral wavelength. Structures consisting liquid organic solutions and air holes has the sensitivity variation of $-2.08 \mathrm{~nm} /{ }^{\circ} \mathrm{C}$ between $25^{\circ} \mathrm{C}-60^{\circ} \mathrm{C}$. Double SMF sandwiched PCF has a metal thin layer and the sensitivity differs 250 $\mathrm{pm} /{ }^{\circ} \mathrm{C}$. All of these structures use these equations beneath,

$$
\begin{aligned}
& \mathrm{n}_{\text {ethanlo }}=1.35265+0.00306 \lambda^{-2}+0.00002 \lambda^{-4} \\
& \mathrm{n}_{\text {chloroform }}=1.431364+0.00563241 \lambda^{-2}-0.00020805 \lambda^{-4}
\end{aligned}
$$

Sensitivity, $S \lambda[n m /(o) C]=\left(\Delta \lambda_{-}\right.$peak $) / \Delta \mathrm{T}$

Figure of merit, $\mathrm{FOM}=\mathrm{S}_{\lambda}\left[\mathrm{nm} /{ }^{\circ} \mathrm{C}\right] / \mathrm{FWHM}(\mathrm{nm})$

Figure of merit is a ratio of sensitivity over wavelength in temperature sensors [1].

Again, using Sellmeier equation, the refractive index sensor follows the mathematical statements as,

$$
\begin{aligned}
& n^{2}(T)=\left(1.31552+6.90754 \times 10^{-6} T\right)+\frac{\left(0.788404+23.5835 \times 10^{-6} T\right) \lambda^{2}}{\lambda^{2}-\left(0.0110199+0.584758 \times 10^{-6} T\right)}+\frac{\left(0.91316+0.548368 \times 10^{-6} T\right) \lambda^{2}}{\lambda^{2}-100} \\
& \text { sensitivity, } S(T)=\frac{d \lambda}{d T}=\frac{\frac{\partial B(\lambda T)}{\partial T} \lambda(T)+\frac{d L}{L d T} B(\lambda, T) \lambda(T)}{B_{g}(\lambda, T)}
\end{aligned}
$$

The sensitivity above is for Bragg grating sensor which are difficult to application criteria [26]

Vibration sensors have sensitivity using butterfly MOF and exercises the relation

$$
\begin{aligned}
& \text { Transfer function, } \mathrm{T}(\delta)=\frac{1}{2}+\frac{1}{2}\left(\delta-\frac{\pi}{2}\right) \\
& \delta_{\text {stress }}=\frac{8 n^{3}}{\lambda E} L_{\text {stress }}(1+\mathrm{v}) \times\left(P_{12}-P_{11}\right) \times\left(\frac{F}{2 b}\right) \\
& \delta_{T}=\frac{2 \pi}{\lambda} \Delta n_{i} L_{T}\left[\beta_{s}+\alpha_{s}\right] \Delta T
\end{aligned}
$$

The parameters here basically depend on wavelength of fiber, PCF length, photoelectric coefficients, Poisson's ratio and stress shiftings.
The corresponding variables and constants are $\lambda, \mathrm{L}, \mathrm{P}, v$ and $\delta$, respectively [17].

\section{Prospects of PCF in Sensing}

PCF sensors have enormous application level, so far seen in this article. The key points in this article are the sensitivity of numerous PCF sensors according to their application field. The results and future aspects can be fetched after the following details so far. The maximum working or functioning level is depicted here.

Increasing sensing range and decreasing sensitivity can be honed using different arrays of cavity radii for a bio sensor. The best sensitivity results for a bio sensor so far is $2200 \mathrm{~nm} / \mathrm{RIU}$ in $1.33 \sim 1.36 \mathrm{RIU}$ and $266 \mathrm{RIU}^{-1}$ with resolution $3.75 \times 10^{-5} \mathrm{RIU}$. Peanut size curvature sensor has the maximum sensitivity $-1.03 \mathrm{~nm} / \mathrm{m}^{-1}$ and $50.5 \mathrm{~nm} / \mathrm{m}^{-1}$ within the range of $2.8 \mathrm{~m}^{-1}$ wavelength. Increasing air hole diameter and decreasing pitch, high relative sensitivity of $48.26 \%$ at the wavelength level $1.3 \sim 2.2$ um is proposed for PCF gas sensors. For a Plasmon sensor, the highest spectral sensitivity is $4500 \mathrm{~nm} / \mathrm{RIU}$. Silver coated gas layer should be enhanced here during COMSOL simulation. For higher birefringence pressure sensor, RI sensitivity is shown as $8.97 \times 10^{-6} / \mathrm{MPa}$ (fast) and $6.48 \times 10^{-6} / \mathrm{MPa}$ (slow). For RI sensor, the resolution defines sensitivity more than anything. Hence for a RI sensor, maximum resolution found so far is $3.9 \times 10^{-5} \mathrm{RIU}$ at the wavelength of $0.36 \mathrm{~cm}$. For a temperature sensing applications of PCF, sensitivity shifts $250 \mathrm{pm} /{ }^{\circ} \mathrm{C}$ in liquid analysis. Also in $1.409 \mathrm{RI}$, the sensitivity differs from $43400 \mathrm{~nm}$ to 77000 $\mathrm{nm}$ per unit RI. Torsion sensors have many areas in sensing applications such as-temperature, strain and resolution. Highest torsion measured so far is up to $22.336 \mathrm{~dB} / \mathrm{rad}$. The temperature sensitivity also measured here as $-2 \times 10^{-4} \mathrm{rad} /{ }^{\circ} \mathrm{C}$ and strain sensitivity as $-6.39 \times 10^{-6} \mathrm{rad} /{ }^{\circ} \mathrm{C}$. The resolution and intensity loss is $0.025^{\circ}$ and $0.0394 \mathrm{~dB} /{ }^{\circ} \mathrm{C}$ respectively.

\section{Conclusion}

This article reviews the recent progress in optical sensors using photonic crystal fiber technology, which is newly adopted beyond conventional optical fibers due to their unique geometric structures. PCFs have special properties. This article will focus the most conversant sensing application areas with their consistent parameters and perception. Numerous photonic crystal fiber (PCF) sensors are available in day-to-day world as well as many are tentative. Researchers and technologists are working on PCFs with more than 15-20 application areas. In this article, sensing applications in physical and bio-chemical with their different types are reviewed recent times. Physical sensors as temperature, curvature, torsion, vibration, pressure, refractive index, evanescent; where the bio-chemical sensors as humidity, gas, $\mathrm{pH}$, liquid etc. has been reviewed in this article paper. The result shows that, most physical sensing applications are varied with their different structures and parameters. Biosensors uses two layers circular lattice with gold layer thickness and the range places between 1.33 to 1.36 refractive indices (RI). Sensitivity are mostly used their unit as refractive index unit (RIU) for different nano-meter scaling. Curvature sensors have the sensitivity $50.5 \mathrm{~nm}$ within the range of $10 \mathrm{~m}^{-1}$ to $22.4 \mathrm{~m}^{-1}$.

\section{References}

1. Yang XC, Lu Y, Liu BL, Yao JQ (2016) Temperature Sensor Based on Photonic Crystal Fiber Filled With Liquid and Silver Nanowires. Tianjin University, Tianjin 300072, China, OIEEE-2016.

2. Ademgil H, Haxha S (2015) PCF based Sensor with high Sensitivity, high birefringence and Low Confinement Losses for Liquid Analyte Sensing Applications. MDPI article.

3. Khan MRR, Kang SW (2016) Highly Sensitive Temperature Sensors Based on 
Citation: Riyadh SMBA, Hossain MM, Mondal HS, Rahaman ME, Mondal PK, et al. (2018) Photonic Crystal Fibers for Sensing Applications. J Biosens Bioelectron 9: 251. doi: 10.4172/2155-6210.1000251

Fiber-Optic PWM and Capacitance variation Using Thermochromic Sensing Membrane. MDPI article.

4. Zan X, Qian L, Wang M, Liu W (2016) The research on temperature sensing properties of photonic crystal fiber based on Liquid crystal filing. MATEC web of Conferences.

5. Liu H, Yang J, Qiao X, Wang Y, Liu X, et al. (2017) Curvature and Temperature Measurement based on a Few-Mode PCF formed M-Z-I and an Embedded FBG. MDPI article.

6. Dash JN, Jha R (2015) PCF Modal interferometer based on macrobending for refractive index sensing. IEEE Sensors Journal.

7. Sun B, Huang Y, Liu S, Wang C, He J, et al. (2015) Asymmetrical in-fiber Mach-Zehnder interferometer for curvature measurement. Optical Society of America.

8. Eliana I. Pacheco-Chac_on, E. Gallegos-Arellano, Juan M. Sierra-Hernandez, Roberto Rojas-Laguna, Julian M. Estudillo-Ayala, Emmanuel Hernandez D, Jauregui-Vazquez, JC, Hernandez-Garcia, "Temperature sensing setup based on an aluminum coated Mach-Zehnder Interferometer", Mexico 2017.

9. Liu C, Wang F, Lv J, Sun T, Liu Q, et al. (2015) Design and theoretical analysis of a photonic crystal fiber based on surface plasmon resonance sensing. Journal of Nanophotonics 9: 2015

10. Dharchana T, Sivanantharaja A, Selvendran S (2017) Design of Pressure Sensor using 2D Photonic Crystal. AENSI Publication.

11. Turduev M, Gidenb IH, Ceren B, Emre B, Boztu ga C, et al. (2017) Mid-infrared T-shaped photonic crystal waveguide for optical refractive index sensing. Elsevier research article.

12. Sahar BE, Myers CR, Armin A, Combes J, Milburn GJ (2015) An integrated quantum photonic sensor based on Hong-Ou-Mandel interference. The University of Queensland, St Lucia, QLD 4072, Australia.

13. Fu HY, Tam HY, Shao LY, Dong X, Wai PKA, et al. (2008) Pressure sensor realized with polarization-maintaining photonic crystal fiber-based Sagnac interferometer. OSA.

14. Rifat AA, Mahdiraji AG, Chow DM, Shee YG, Ahmed R, et al. (2015) Photonic Crystal Fiber-Based Surface Plasmon Resonance Sensor with Selective Analyte Channels and Graphene-Silver Deposited Core. Sensors 15: 1149911510.
15. Huang B, Shu X, Du Y (2017) Intensity modulated torsion sensor based on optical fiber reflective Lyot filter. Optics Express research paper.

16. Hu T, Zhao Y, Wu D (2015) Novel torsion sensor using a polarization maintaining photonic crystal fiber loop mirror. Instrumentation Science and Technology.

17. Chah K, Linze N, Caucheteur C, Mégret $P$, Tihon $P$, et al. (2012) Temperatureinsensitive polarimetric vibration sensor based on $\mathrm{HiBi}$ microstructured optical fiber. OSA Journal.

18. Morshed M, Hassan MI, Roy TK, Uddin MS, Razzak ASM (2015) Microstructure core photonic crystal fiber for gas sensing applications. Applied Optics Journal 54: 8637-43.

19. Bykov DS, Schmidt OA, Euser TG, Russell PSJ (2015) Flying particle sensors in hollow-core photonic crystal fiber. Nature Photonics.

20. Kassani SH, Khazaeinezhad R, Jung Y, Kobelke J, Oh K (2015) Suspended Ring-Core Photonic Crystal Fiber Gas Sensor With High Sensitivity and Fast Response. IEEE Photonics Journal.

21. Asaduzzaman S, Ahmed K, Bhuiyan T (2016) Design of simple structure Gas sensor based on Hybrid Cladding Photonic Crystal Fiber. Cumhuriyet University Faculty of Science Journal.

22. Hasan RM, Akter S, Rifat AA, Rana S, Ali S (2017) A Highly Sensitive Gold-coated Photonic Crystal Fiber Biosensor Based on Surface Plasmon Resonance. Photonics.

23. Zolkapli M, Saharudin S, Herman SH, Abdullah WFH (2016) A Numerica Analysis of Various $\mathrm{pH}$ Level for Fiber Optic $\mathrm{pH}$ sensor Based on Bromophenol Blue in Silica. IJEESR Journal.

24. Ascorbe J, Corres JM, Arregui FJ, Matias IR (2017) Recent Developments in Fiber Optics Humidity Sensors. MDPI review.

25. Asaduzzaman S, Ahmed K, Paul BK (2016) Slotted-core Photonic Crystal Fiber in Gas Application", Advanced Sensor Systems and Applications.

26. Liu Q, Li SG, Chen H (2017) Enhanced Sensitivity of temperature sensor by a PCF with a defect core based on Sagnac Interferometer. Sensors and Actuators Journal.

27. Xu W, Yao J, Yang X, Shi J, Zhao J, et al. (2016) Analysis of Hollow Fibe Temperature Sensor Filled with Graphene-Ag Composite Nanowire and Liquid. Sensors.

28. https://www.techopedia.com/definition/24948/photonic-crystal-fiber-pcf 